\title{
Bandwidth Reservation in Mobile Adhoc Networks
}

\author{
Redouane Belbachir, \\ Mekkakia Maaza Zoulikha, \\ belbachir_red@yahoo.fr,_zoulikh@gmail.com,_kies_ali@yahoo.fr, \\ Department of Mathematics and Computer Science, \\ University of Oran Mohamed Boudiaf-USTO, Algeria.
}

\author{
Bernard Cousin \\ bcousin@irisa.fr \\ IRISA \\ University of Rennes 1, France.
}

\begin{abstract}
The bandwidth reservation is one of most adopted solutions to meet QoS requirements in 802.11 ad hoc networks. The efficiency of these solutions depends on the accuracy of their estimations of available bandwidth; otherwise, their application can be catastrophic on networks. Therefore, accurate bandwidth estimation is fundamental, where each networks characteristic must be taken into consideration, including mobility and medium sharing. Current solutions do not take into account all networks characteristics, resulting to wrong bandwidth estimations and QoS violations. In this paper, we present a new approach for bandwidth reservation -Accurate Bandwidth Reservation (ABR)which embeds an improved method of available bandwidth measurement, where all criteria of such networks are considered. Evaluation of $A B R$ is performed by simulations and comparisons with some existing approachs.
\end{abstract}

Index Terms-Ad hoc networks, Accuracy, Mobility, Bandwidth Estimation, ABR.

\section{INTRODUCTION}

I EEE 802.11-based ad hoc networks are able to provide some QoS level through an service differentiation, due to the IEEE 802.11e amendment. However, no solution has been standardized for reservation of critical ressources like bandwidth. The reservation of communication resources like bandwidth becomes necessary for QoS flows guarantees, in order that flows are delivered without that there bandwidth is degraded, and so avoid delaying delivering or data losses.Thus, the reservation must be accurate enough to assure the admissions of right flows. Several solutions for bandwidth reservation have been proposed, which results to failures reservations because of their inaccurate available bandwidth (AB) estimation methods.

The performance of BR solutions is evaluated by two metrics "false admission rate" and "false reject rate". False admission means that flows whose bandwidth consumption is beyond the capacity of the network are admitted. The cause of false admission, is the overestimation of $\mathrm{AB}$. False reject means that flows whose bandwidth consumption is not beyond the capacity of the network are rejected, caused by the $A B$ underestimations.

In this paper, we present a novel bandwith reservation approach, named ABR (Accurate Bandwidth Reservation), which provides accurate admission control for bandwidth reservation in mobile ad hoc networks, by improving the available bandwidth estimations. We noticed that the estimation errors are due to two items. The first one is the neglect of some network criteria, and the second is a bad computing and/or integration of the considered network criteria in estimations.
ABR increases the accuracy of available bandwidth estimation by considering each wireless 802.11 ad hoc network criteria as the overlap of the channel idle periods, collisions and mobility.

\section{RELATED WORK}

$\mathrm{AB}$ between two neighbor nodes, is defined as the maximum throughput that can be transmitted between these two nodes without degrading the throughputs of existing flows in the network. In order to evaluate AB in wireless ad hoc networks, some authors [1] adopt methods which are intended firstly for wired networks [2], where measurements are based on the injection of probe packets. These methods were inefficient in wireless IEEE 802.11 networks since the large inaccuracy because of channel access proprieties. Therefore, there is a reorientation towards a measurement methods based on the passive monitoring of the networks activities. The authors of QoS-aware [3] routing protocol, estimate $\mathrm{AB}$ per node, by updating periodically the rate value of the channel availability time sensed at MAC layer. So the available bandwidth $A B_{i}$ on node " $i$ " is defined as:

$$
A B_{i}=\frac{t_{i}}{\Delta} \cdot C=I_{i} \cdot C
$$

Where $t_{i}$ is the channel availability time, sensed at node " $i$ " during the period $\Delta, C$ is the channel capacity and $I_{i}=\left(t_{i} / \Delta\right)$ is the channel availability rate.

Since the contention for medium access due to its sharing between nodes, the bandwidth is also shared. So, identify the medium availability at node must be joined by the medium availability at its neighbors. In QoS-AODV [4], the authors use the exchange of "hello" packets to exchange the computed $\mathrm{AB}$ per node in neighborhood. Then, $\mathrm{AB}$ at one node is considered as the minimum $\mathrm{AB}$ of its neighborhood. Some papers consider the bandwidth sharing on carrier sense range, such as BRuIT [5], CACP [6] and IAB [7]. In BRuiT, each node provides information about its $A B$, and about its neighbors. So the admission control is based on two-hop neighborhood knowledge, In CACP, the node's transmission power is increased during the "hello" packet exchange, so AB per node information reaches nodes on carrier sensing range. And in IAB, an probabilistic method is used. All the proposed methods of bandwidth estimation share the same principle with $\mathrm{AAC}[8]$ for $\mathrm{AB}$ definition on one link, as : $\left(A B_{s, r}\right.$ is the $\mathrm{AB}$ on link $(s, r)$ )

$$
A B_{s, r}=\min \left\{A B_{s}, A B_{r}\right\}
$$




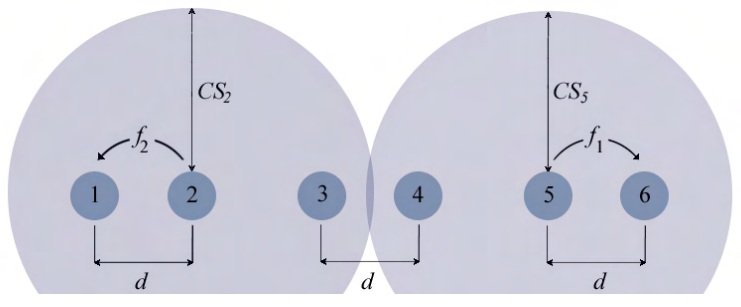

Fig. 1. Scenario illustrating the problem of idle time overlaps

\section{ABR APPROACH}

The aim of the BR solutions is to ensure the data flow delivery without bandwidth degradation, while maintaining the throughputs of the ongoing flows in network. Given the features of the 802.11-based mobile ad hoc networks, the accurate $\mathrm{AB}$ estimation is a critical challenge that is addressed in this section.

\section{A. The overlap of the channel idle periods}

For brevity, $N_{i}$ and and $(i, j)$ are the abbreviation of Node $i$ and wireless link between node $N_{i}$ and $N_{j}$ respectively. Consider the topology of 6 nodes disposed as depicted in Fig. 1. The distance $d$ is the communication radius, $C S_{i}$ is the sensing radius of $N_{i}$. Therefore, $N_{4}$ (respectively $N_{3}$ ) senses the communication between $N_{5}$ and $N_{6}$ (respectively between $N_{1}$ and $N_{2}$ ). Flows $f_{1}$ and $f_{2}$ which are established on links (1, 2 ) and $(5,6)$ respectively, have same and constant bandwidth consuming about $50 \%$ of medium capacity. We intend to evaluate the $\mathrm{AB}$ on link $(3,4)$ according the bandwidth consumed by flows $f_{1}$ and $f_{2}$. Let's the two scenarios cases :

1) Sources $N_{2}$ and $N_{5}$ start exactly at the same time to transmit their flows. The two transmissions are completely synchronous.

2) $N_{2}$ sends each data packet exactly after that $N_{5}$ receives the acknowledgment of its data packet (MAC layer ACK). The two transmissions are completely asynchronous.

In both scenarios, the values of medium's idle time at nodes $N_{3}$ and $N_{4}$ are similar about $50 \%$. The difference between both scenarios, is the shift between idle times. In first scenario, the periods of medium availability of both nodes overlap, offering several communication opportunities, which explains the real $\mathrm{AB}$ of link $(3,4)$ is about $50 \%$, with decreasing sometimes to $40 \%$ (because of the medium collisions). This scenario confirms the accuracy of estimation methods based on formula (2), particularly methods that consider collisions like IAB solution. In second scenario, the $\mathrm{AB}$ on link $(3,4)$ is practically null. The periods of medium availability of both nodes never overlap. When the medium is available at $N_{3}$, it is not available at $N_{4}$. So communication opportunities never exist during this scenario, although the medium is idle at $50 \%$ of time at $N_{3}$ and also at $N_{4}$.

So, the link's $\mathrm{AB}$ depends on the overlap between the medium idle periods at transmitter and receiver. Evaluating the impact of this overlap requires an fine mechanism of clock synchronization and huge overhead due to aditional

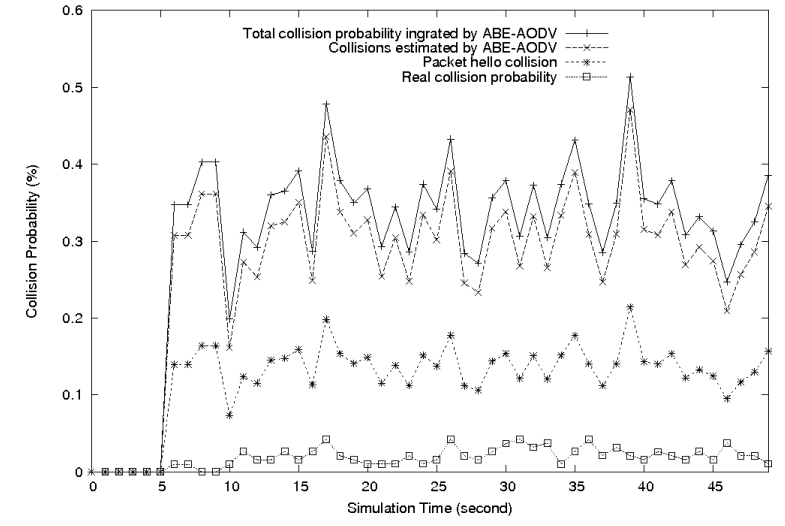

Fig. 2. Collision probabilities

informations exchange. Therefore, we use the probabilistic average time to estimate the effect of this phenomenon.

Since communications on links $(5,6)$ and $(2,1)$ are totaly independent, the channel idle periods on $N_{3}$ and $N_{4}$ are also independent. So, the average of communication opportunities is the average times where the channel idle periods on nodes overlap. Let's $S_{s, r}$ the average of communication opportunities between $N_{s}$ and $N_{r}$. We notice : $S_{s, r}=S_{s} \cap S_{r}$, where $S_{i}$ is the set of channel's idle periods at $N_{i}$. By considering that distribution of the channel's idle periods at $N_{s}$ and $N_{r}$ are totally independent, the average time of communication oppotunities is : $\left(I_{r} \cdot I_{s}\right)$. And the $\mathrm{AB}$ on wireless link (s,r) is defined as:

$$
A B_{s, r}=\left(I_{r} \cdot I_{s}\right) \cdot C
$$

ABE-AODV [9] uses this same definition, but, it is an conservative solution because of the method by which it calculates and integrates the collisions phenomena in its bandwidth estimations.

\section{B. Collisions on 802.11-based Ad Hoc Networks}

Considering the overlap between the channel's idle periods at sender and receiver is not sufficient to decide about the link ability to convey a flow. Indeed, the collisions problems must be considered accurately, otherwise it conducts to erroneous estimates. ABE-AODV method computes $\mathrm{AB}$ on link (s,r) as $A B E_{-} A O D V_{(s, r)}=(1-K) \cdot A B_{s, r} \cdot(1-P)$. Where $K$ is the rate consumed due to the backoff time when collisions happen. $P$ is the collision probability. ABE-AODV underestimates the $\mathrm{AB}$ issue, because of the excessive presence of collision rate in the computing formula (which already has the backoff $(K)$, and the collision probabilities $(P)$ ), and because of the method used to compute the collision probability.

When "hello" packets are issued regularly, in some methods, the receiver estimates the amount of these packets that it should receive in a given time interval. Comparing this figure with the actual number of "hello" packets received gives an estimate of the probability of collision between two peers. This confuses the effects of congestion-related losses due to collisions. When a node does not succeed in emitting as many "hello" packets as it should (due to an overloaded medium). Its neighbors considers that there are collisions 


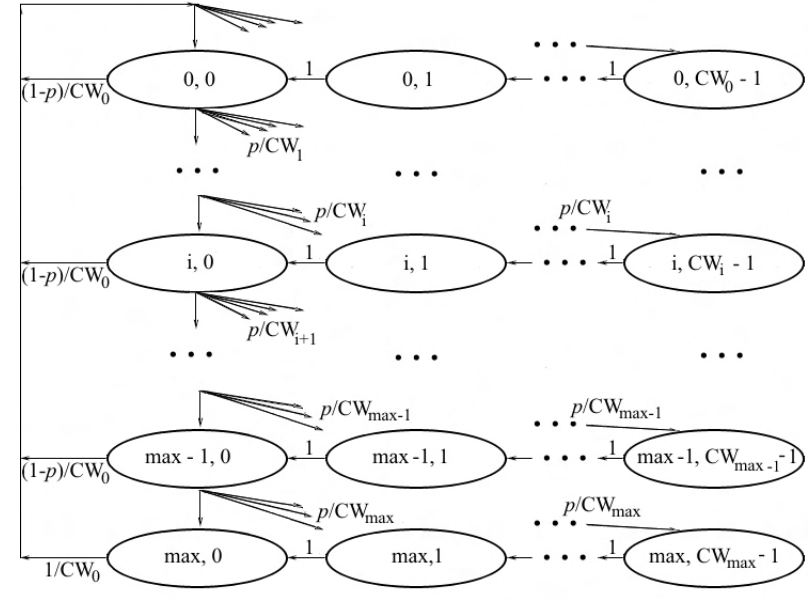

Fig. 3. Markov Chain model for the backoff window size.

while there is not. ABE-AODV uses this collision probability "Phello", and defines the probability of data packet collision (of 1000 bytes, data's size) as: $P=P_{\text {hello }} \cdot 2.19$. We conduct a simulation on random topology of 30 nodes on area $(900 \mathrm{~m} \cdot 500 \mathrm{~m}) .2$ CBR flows, each flow is from one different source to one different destination are established. Fig. 2. represents the real collision probabilities of data packets compared to the estimated $P_{\text {Hello }}$ values and the total collision probability estimated by ABE-AODV. Note that following $P_{\text {Hello }}$ values, the collisions probabilities are overestimated, but the calculation and integration methods used by ABEAODV, lead to an even worse estimations. Therefore, this means also a large underestimations of $\mathrm{AB}$. In ABR approach, the conditional probability [10] is used. $P_{i}$ is the conditional collision probability on $N_{i}$ which has $n$ neighbor nodes. $P_{i}=\tau_{i} \cdot\left[1-\prod_{j=0}^{n}\left(1-\tau_{j}\right)\right]$. Where $\tau_{j}$ is the transmission rate by $N_{j}$.

During the transmission from sender $N_{s}$ to $N_{r}$, the packets may have collisions at $N_{s}$ or $N_{r}$. If there was, in both cases the exponential backoff mechanism is triggered only at $N_{s}$. Knowing that during the backoff time a node cannot transmit even the medium is idle [13]. So, there is an proportion of idle time which could be lost. If we consider an average backoff time at $N_{s}$ is $\overline{\text { backoff }}$, the $\mathrm{AB}$ on " $N_{s}$ " becomes:

$$
A B_{s}=\frac{\left(\left\lfloor t_{s}-\overline{\text { backoff }}\right\rfloor\right)}{\Delta} \cdot C
$$

Note, that during the backoff time, a node can receive packets (the backoff is said to be frozen and it resumes after reception). So, the backoff mechanism activation has no effects on the receiver node. Therefore, the $\mathrm{AB}$ on the link $(\mathrm{s}, \mathrm{r})$ can be estimated more precisely as:

$$
A B_{s, r}=I_{r} \cdot \frac{\left(\left\lfloor t_{s}-\overline{b a c k o f f_{s}}\right\rfloor\right)}{\Delta} \cdot C
$$

Initially, the backoff mechanism uniformly selects an value in the interval $\left[0, C W_{0}-1\right],{ }^{1}$ where $C W_{0}$ is the minimal

\footnotetext{
${ }^{1}\left[0, C W_{0}-1\right]$ is the first contention range, each value represent a slot time's number, it is defined by MAC protocol specifications.
}

contention window. The backoff timer decrements the value selected. When the timer reaches 0 it transmits the packet.

This decrementation's procedure is represented by the stochastic process $b(t)$ where " $t$ " represents the contention range values. When collisions happen, the exponential backoff mechanism is triggered. After each unsuccessful transmission, the contention window size is doubled up to a maximum value denoted by $C W_{\max }=2^{\max } \cdot C W_{0}$, or until successful tranmission. Thus in the Markow model, there is transition from one contention range to another, where $\max$ represents the last stage. And let consider $s(t)$ the stochastic process representing the backoff stage at slot time $t$. The bidimensional backoff process $\{s(t), b(t)\}$ is modeled with the discrete-time Markov chain depicted in Fig. 3. An markov model that is similar to Bianchis markovian model [12]. The difference between the two models is onto the last stage of the backoff. Following Bianchis model, the backoff will be absolutly not initialized, as long as an packet is not successfully transmitted. Other side, the depicted model in Fig. 3 considers that when last backoffs stage is achieved then there will be return to the first stage (by taking another packet or the same). In this new Markov chain, the only non null transition probabilities are:

$$
\begin{cases}P\{i, k \mid i, k+1\}=1 & k \in\left(0, C W_{i}-2\right) \\ & i \in(0, \max ) \\ P\{i+1, k \mid i, 0\}=\frac{p}{C W_{i+1}} \quad & k \in\left(0, C W_{i+1}-1\right) \\ & i \in(0, \max -1) \\ P\{0, k \mid i, 0\}=\frac{1-p}{C W_{0}} & k \in\left(0, C W_{0}-1\right) \\ & i \in(0, \max -1) \\ P\{0, k \mid \max , 0\}=\frac{1}{C W_{0}} & k \in\left(0, C W_{0}-1\right)\end{cases}
$$

Where :

$P\left\{i_{1}, k_{1} \mid i_{0}, k_{0}\right\}=P\left\{s(t+1)=i_{1}, b(t+1)=k_{1} \mid s(t)=\right.$ $\left.i_{0}, b(t)=k_{0}\right\}$

Let consider the stationary distribution of the chain :

$b_{i, k}=\lim _{t \rightarrow \infty} P\{s(t)=i, b(t)=k\}$

Owing to the chain regularities:

$$
\begin{gathered}
b_{i, k}=\frac{C W_{i}-k}{C W_{i}} \cdot \begin{cases}b_{\max , 0}+(1-p) \cdot \sum_{j=0}^{\max -1} b_{j, 0} & i=0 \\
p \cdot b_{i, 0} & 0<i \leq \max \end{cases} \\
b_{i, 0}=p \cdot b_{i-1,0}=p \cdot p \cdot b_{i-2,0}=\cdots=p^{i} \cdot b_{0,0} \\
\Rightarrow b_{i, 0}=p^{i} \cdot b_{0,0}
\end{gathered}
$$

Knowing that the probabilities sum is 1 , then : 


$$
\begin{aligned}
& \sum_{i=0}^{\max } \sum_{k=0}^{C W_{i}-1} b_{i, k}=1 \\
& 1=b_{0,0} \cdot\left[\sum_{k=0}^{C W_{0}-1} \frac{C W_{0}-k}{C W_{0}}+\sum_{i=1}^{\max } p^{i} \cdot \sum_{k=0}^{C W_{i}-1} \frac{C W_{i}-k}{C W_{i}}\right] \\
& b_{0,0}=2 \cdot\left[C W_{0}+1+p \cdot\left(\frac{1-p^{\max }}{1-p}+2 C W_{0} \cdot \frac{1-(2 p)^{\max }}{1-(2 p)}\right)\right]^{-1}
\end{aligned}
$$

The average backoff time Pbt on one node to transmit one packet with collision probabilty $p$, is defined as:

$$
\begin{aligned}
& P b t=\sum_{i=0}^{m} \sum_{k=0}^{C W_{i}-1} k \cdot b_{i, k} \\
& \text { Then : } \\
& P b t=\frac{b_{0,0}}{6} \cdot\left[C W_{0}^{2}-1+p \cdot\left(\frac{4 C W_{0}^{2}\left(1-(4 p)^{\max }\right)}{1-4 p}-\frac{1-p^{\max }}{1-p}\right)\right]_{(7)}
\end{aligned}
$$

So, on the measurement interval time $\Delta$ for node $s$ :

The average backoff time $\overline{\text { backof } f_{s}}$ on the measurement interval time $\Delta$ at $N_{s}$, is :

$$
\overline{\text { backof } f_{s}}=[(\text { Pbt } \cdot \text { Slot })+\text { DIFS }] \cdot \frac{\tau_{s} \cdot C}{\text { Psize }}
$$

Where Slot is the slot time size [13] considered in this paper as $20 \mu s, D I F S$ [13] is fixed to $50 \mu s$, and Psize is the data packet size.

\section{Mobility Management in Measures}

Increasing the exchange rate of "hello" packets is the solution mostly used in order to reduce the mobility impact. Let's the wireless link (s,r), with medium's capacity of $2 \mathrm{Mb} / \mathrm{s}$. The mobility leads to an instability of distance between $N_{s}$ and $N_{r}$. Suppose there is no traffic on the network and the distance between the two nodes is larger than communication radius, until time $\lambda_{1}$, each node becomes in the communication radius of the other. Fig. 4. shows the link's state during two consecutive measurement periods $\Delta_{1}$ and $\Delta_{2}$. In first period, the link exists only during $\mathrm{X}=10 \%$ of $\Delta_{1}$ period. At $T$, all bandwidth measures give the same wrong value of $A B_{s, r}$, about $100 \%$ of availability. By considering that ( $\Delta_{1}=\Delta_{2}=1$ second), means that up to $1.6 \mathrm{Mb}$ of traffic ${ }^{2}$ could be transmitted during $\left[\lambda_{1}, T\right]$ period, means 0.1 second. To avoid this wrong, the proportion indicated by $X$ in Fig. 4. must be considered in measures. The consideration is crucial particularly when the admission control is executed at $T$ and the proportion $X$ is in the measurement period that follows, as shown in Fig. 4 . by the interval $\left[T, \lambda_{2}\right]$. Therefore, the equation (7) became :

$$
A B_{s, r}=I_{r} \cdot \frac{\left(t_{s}-\overline{\text { backof } f_{s}}\right)}{\Delta} \cdot C \cdot \frac{\lambda_{2}-T}{\Delta}
$$

In order to compute $\lambda_{2}$ value at $T$ or before, we use an mobility prediction method, similar to that presented in [12]. Considering that each node $s$ (respectively $r$ ) moves at velocity $V_{s}$ (respectively $V_{r}$ ), with direction $\alpha$ (respectively $\beta$ ). $t_{j}$ value is calculated as (for example at node $s$ ):

\footnotetext{
${ }^{2} 2 \mathrm{Mb} / \mathrm{s}$ of medium capacity and 1000 bytes of data packets size, result to $1.6 \mathrm{Mb} / \mathrm{s}$ application layer throughput.
}

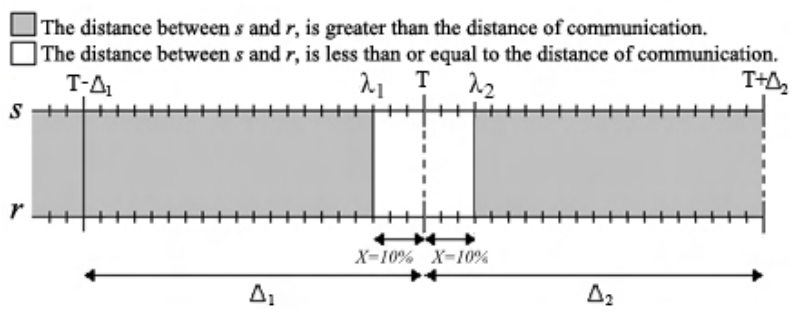

Fig. 4. Link status during a $(\Delta)$ measurement period.

$$
\lambda_{2}=\left\{\begin{array}{l}
\infty \text { if }\left(V_{s}=V_{r} \text { and } \alpha=\beta\right) \\
\frac{-(e f+g h)+\sqrt{\left(e^{2}+g^{2}\right) \cdot d^{2}-(e h-g f)^{2}}}{\left(e^{2}+g^{2}\right)} \text { else }
\end{array}\right.
$$

Knowing that $\left(x_{s}, x_{r}\right)$ and $\left(y_{s}, y_{r}\right)$ are the coordinates of nodes $s$ and $r$ resepectively. And by considering :

$$
\begin{aligned}
& e=\mathrm{V}_{s} \cdot \cos (\alpha)-V_{r} \cdot \cos (\beta) \\
& f=x_{s}-x_{r} \\
& g=\mathrm{V}_{s} \cdot \sin (\alpha)-V_{r} \cdot \sin (\beta) \\
& h=y_{s}-y_{r}
\end{aligned}
$$

The coordinates are given through GPS system. During the exchange of "hello" packets, each node indicates the starting coordinates of its motion and the coordinates of its destination, as well the speed of its motion. Through these, the receiving nodes of the "hello" packets, compute the motion direction of the "hello" packet's source node .

\section{Protocol Design}

Each node monitors the idle medium rate, its own transmission rate, and caculates the proportion rate which can be consumed by the backoff. When the source has a data flow, it checks firstly the availability of residual bandwidth through equation (1). If the check is positive, it broadcasts an route request packet (RREQ). The source indicates into the RREQ, the bandwidth required for its flow. When node receives the RREQ packet, it performs the admission control by comparing the bandwidth required by source and the estimated available bandwidth on the link constituted with its predecesor node, by using equation (9). If this check is negative discards the RREQ.

In ABR, an node monitors the distances evolution by using the neighborhood's table. The prediction of link failure caused by the mobility, reduces the bandwidth availability following equation (9). If the prediction was after the flow admission, and the predicted time is reached or approached, the node sends a route error packet (RERR) to the source in order to stop its transmissions and avoid losses.

\section{EVALUATION}

We perform an evaluation through simulations by using (NS2). An comparative study is presented in this section, between the simulations's results when ABR, AODV, ABEAODV and IAB solutions are enabled. The medium access 


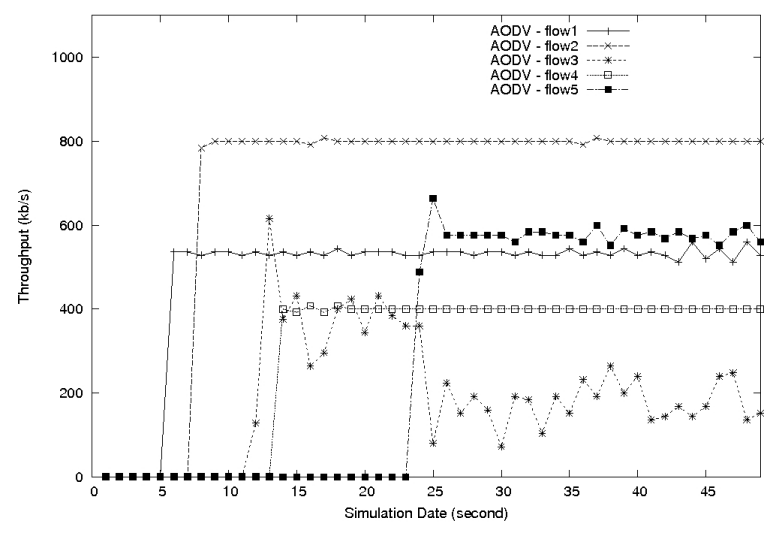

(a) AODV

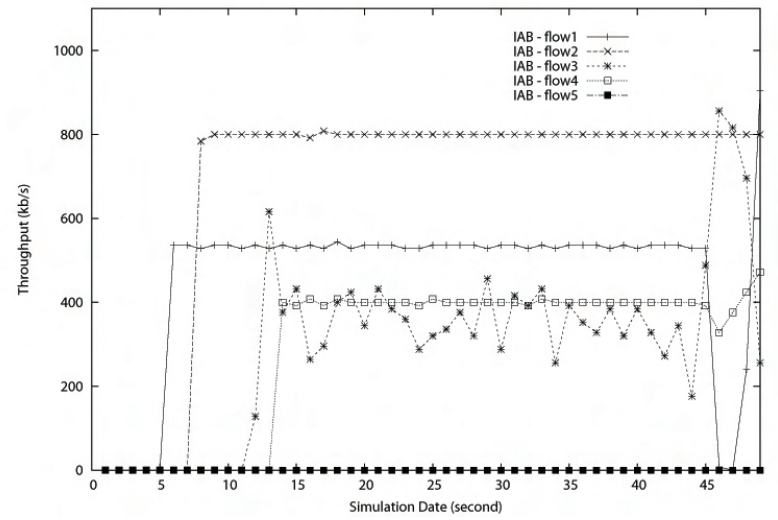

(b) IAB

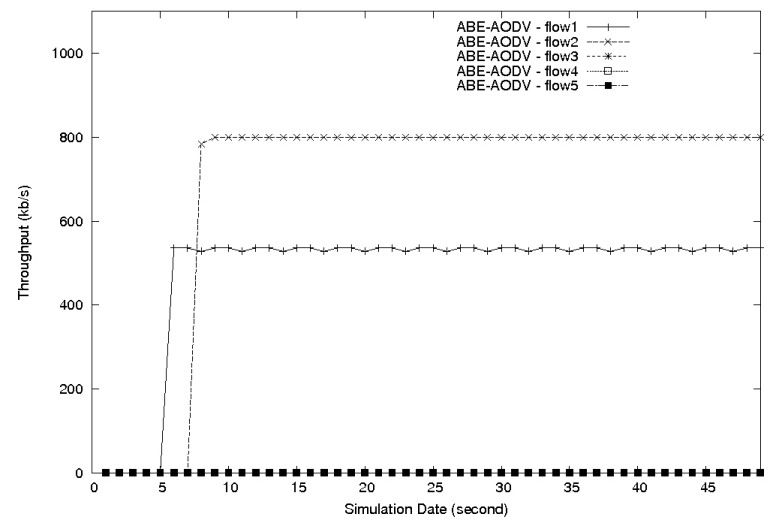

(c) ABE-AODV

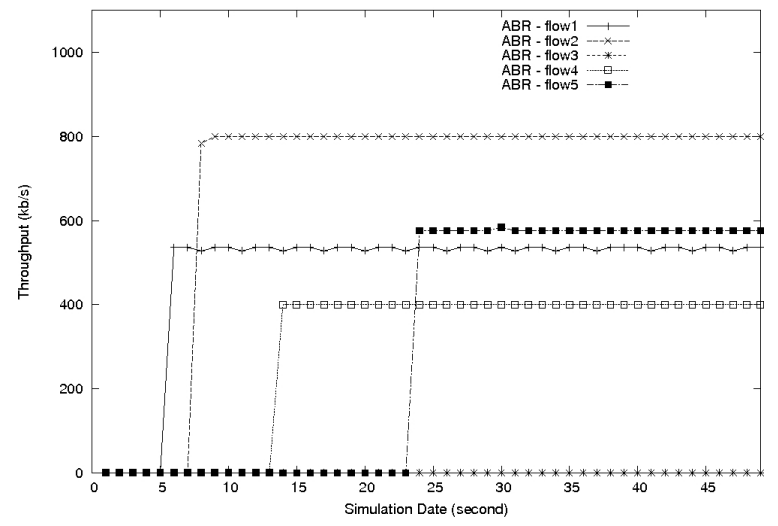

(d) ABR

Fig. 5. Throughputs of flows obtained by (a)AODV, (b)IAB, (c)ABE-AODV, and (d)ABR scheme used is CSMA, the medium capacity is set to $2 \mathrm{Mb} / \mathrm{s}$, and 1000 bytes of data packet's size. The below graphs are the average results of several simulations, where different parameters are considered as trafic load, transmition times, networks densities and mobilities.

\section{A. Accurate estimation in dense networks}

To illustrate the accuracy's estimations of ABR, firstly, an simulation is performed in a $900 \mathrm{~m} \times 500 \mathrm{~m}$ static network with 60 nodes. The nodes are randomly positioned. Five connections of CBR sources are attempted to be established in the network.

Fig. 5(a) represents the throughput evolution of the five flows when no admission control is performed. The network becomes congested by the beging transmission of flow3. As shown, the appearence of flow5 results in dramatical increasing of flow3's throughput.

Fig. 5(b) represents the evolution of the different flows throughputs when IAB is enabled. IAB tends to overestimate the available bandwidth. Thus, admission control mechanism is not enough accurate when flow 3 is accepted, arising an admission in degraded and disrupted state of its throughput. This disruption affects in turn at $45^{t} h$ second the flows (flow2 and flow4), by degrading dramatically the throughputs of these last.

When ABR approach is enabled, all flows are admitted except the second one (flow2). Thus, flow's throughputs are stable as it appeares on Fig. 5(c), all four remaining flows are able to fit into the network, indicating that, for other scenarios, the fourth flow was the only cause of wireless links overload.

However, ABE-AODV tends to underestimate the available bandwidth. On Fig. 5(d), we notice that ABE-AODV accepts only the two first flows among the five. Therefore, admission control mechanism is very severe, resulting in under exploitation of bandwidth in network. In this scenario, underestimations are due to the collision probability's calculation method and its integration manner in bandwidth measurements by ABE-AODV.

\section{B. Mobility management during reservations}

To investigate the mobility effect on the solutions of bandwidth reservations, another simulation is performed on mobile environment. Below, an comparatif description is presented of results achieved by ABR, ABE-AODV and AODV. The simulation modelises 30 mobile nodes randomly positionned. The random way point mobility's model is chosen for the motion of nodes, with speed of $20 \mathrm{~m} / \mathrm{s}$ at maximum. Five CBR flows are generated, throughput's loads are randomly drawn between $300 \mathrm{~kb} / \mathrm{s}$ and $600 \mathrm{~kb} / \mathrm{s}$.

As shown on Fig. 6(a), where no admission control is applied. Once the flow4s transmission has started, the network ends up dramatically congestioned. An high irregularities appear on flows throughputs. The medium is overloaded and all flows suffer. This confirms that medium is not able to sustain all five flows.

In opposite, when ABR's approach is enabled all flows throughputs are stable as shown on Fig. 6(b). This means 


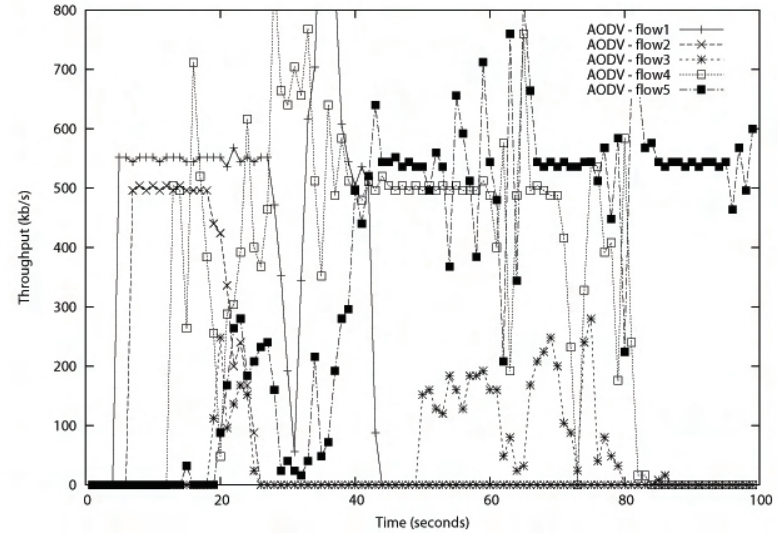

(a) AODV

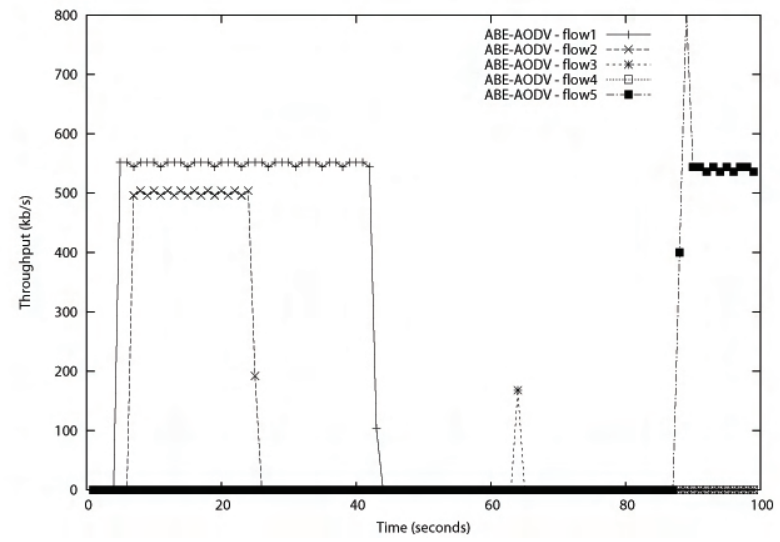

(b) ABE-AODV

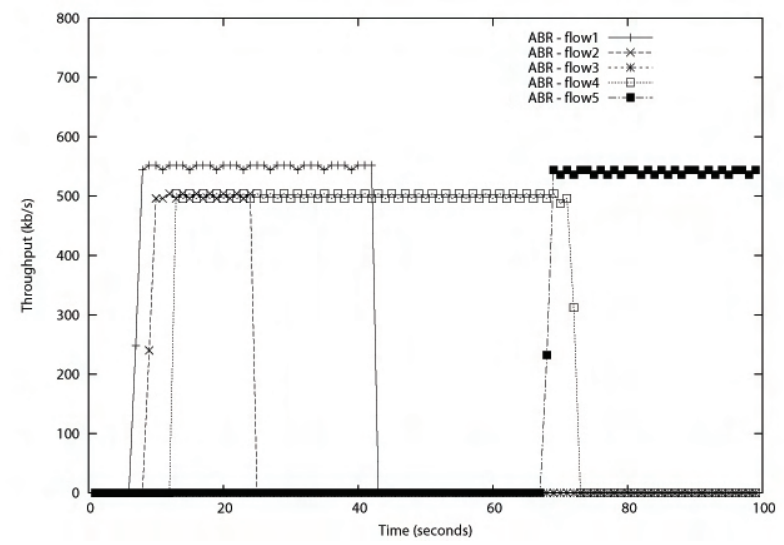

(c) $\mathrm{ABR}$

Fig. 6. Throughputs obtained by (a)AODV, (b)ABE-AODV, and (c)ABR in mobile networks.

that all admitted flows are able to fit into the network. Abr admits all flows when there paths are available and also the bandwidths are sufficient. In except, flow3 is rejected because its path was on breaking.

Fig. 6(b) shows that ABE-AODV fails to perform an accurate admission control. Since, the mobility neglect in bandwidth measures, ABE-AODV allows the admission of flow3 on broken path. ABE-AODV is also too severe with flows, where only the flows 1,2 and 5 are admitted. This little consumption is due to the underestimations the available bandwidth.

\section{CONClusion}

In this paper, we have presented a new approach "ABR" for bandwidth reservation on mobile ad hoc networks. ABR is an improvement of some existing approaches of available bandwidth estimation on wireless links. The main contribution of this research is the right combination during the integration of the phenomena of collision, mobility and the asynchrony of idle's medium periods in the measures available bandwidth.

The ABR's performance is shown through simulation results, and comparative analysis with some existing approaches. The comparison has been particularly with ABE-AODV's approach which is the only with $\mathrm{ABR}$, that consider the asynchrony of idle's medium period. ABR outperforms these approches in the stability of throughputs of flows and bandwidth resource quantities using. Therefore, an more bandwidth exploitation while respecting the required and admitted QoS.

\section{REFERENCES}

[1] A. Johnsson, B. Melander, and M. Bjorkman, Bandwidth Measurement in Wireless Network. In Proceedings of the Fourth Annual Mediterranean Ad Hoc Networking Workshop, Porquerolles, France, June, 2005.

[2] B. Melander, M. Bjorkman, and P. Gunningberg, A New End-to-End Probing Analysis Method for Estimating Bandwidth Bottlenecks. In Proceedings of the Fifth Global Internet Symp. (Global Internet) held in conjunction with Global Comm. Conf. (GLOBECOM 00), Nov. 2000.

[3] L. Chen, and W. Heinzelman, QoS-aware Routing Based on Bandwidth Estimation for Mobile Ad Hoc Networks. IEEE Journal on Selected Areas of Communication, 3, 2005.

[4] R. Renesse, M. Ghassemian, V. Friderikos, and A. H. Aghvami, QoS Routing over Ad hoc On-demand Distance Vector Routing Protocol. Proceedings of IEE 3G Wireless 2004 Conference, London, UK, October 2004.

[5] C. Chaudet, and I. G. Lassous, BRuIT - Bandwidth Reservation under InTerferences influence. Proceedings of European Wireless 2002 (EW2002), Florence, Italy, Feb. 2002.

[6] Y. Yang and R. Kravets, Contention Aware Admission Control for Ad Hoc Networks. IEEE Trans. Mobile Computing, vol. 4, pp. 363-377, 2005.

[7] H. Zhao, E. Garcia-Palacios, J. Wei, and Y. Xi, Accurate available bandwidth estimation in IEEE 802.11-based ad hoc networks. Computer Communications, Issue 32, pp. 10501057, 2009.

[8] R. de Renesse, M. Ghassemian, and V. Friderikos, and A.H. Aghvami, Cross-layer cooperation for accurate admission control decisions in mobile ad hoc networks. IET Communications 1, pp. 577586, 2007.

[9] C. Sarr, C. Chaudet, G. Chelius and I. G. Lassous, Bandwidth Estimation for IEEE 802.11-based Ad Hoc Networks. IEEE Transactions on Mobile Computing, Vol. 7, Num. 10, 2008.

[10] A. Nafaa, Provisioning of multimedia services in 802.11-based networks: facts and challenges. IEEE Wireless Communications 14 (5), pp. 106112, 2007.

[11] R. Belbachir, Z. M. Mekkakia, and A. Kies, Available Bandwidth Estimation with Mobility Management in Ad hoc Networks. Proceedings of The Third International Conference on Wireless, Mobile Network \& Applications (WiMoA/ICCSEA 2011), Dubai, 2011. Springer, CCIS series, Vol. 154, pp. 304-315, 2011.

[12] S. Lee, W. Su, and M. Gerla. Ad hoc wireless multicast with mobility prediction. Computer Communications and Networks, 4-9, 1999.

[13] IEEE Computer Society LAN MAN Standards Committee, Wireless LAN Medium Access Protocol (MAC) and Physical Layer (PHY) Specification, IEEE Std 802.11-1997. The Institute of Electrical and Electronics Engineers, New York, 1997. 